\title{
AN EVALUATION OF NMR CRYOPOROMETRY, DENSITY MEASUREMENT AND NEUTRON SCATTERING METHODS OF PORE CHARACTERISATION
}

\author{
J.B.W.Webber, J.H. Strange and J.C. Dore
}

October 6, 2000

School of Physical Sciences, University of Kent, Canterbury, U.K.

\begin{abstract}
Sol-gel silicas with nominal pore diameters ranging from $25 \AA$ to $500 \AA$ were studied by NMR cryoporometry, and by neutron diffraction and small angle scattering from dry silicas over the $\mathbf{Q}$ range $8 \cdot 10^{-4} \AA^{-1} \leq \mathbf{Q} \leq 17 \AA^{-1}$. Density and imbibation experiments were also performed. Geometric models of porous systems were constructed and were studied by both analytic techniques and Monte-Carlo integration. These models, combined with the information from the above measurements, enabled the calculation of the fully density corrected solid-solid density correlation functions G(r) for the sol-gel silicas, deduction of the (voidless) silica matrix density, measurement of the silica fraction in the grain and of the packing fraction of the silica grains and an estimation of the water equivalent residual hydrogen on the dried silica surface. In addition, the pore diameter $\mathrm{D}$, pore diameter to lattice spacing ratio $\mathrm{D} / \mathrm{a}$, and pore and lattice variance $\sigma$ could also be measured. While the NMR cryoporometry pore diameter measurements for the sol-gel silicas show excellent co-linearity with the nominal pore diameters as measured by gas adsorption, and the calculated pore diameters from the measured neutron scattering show surprisingly good agreement with these measurements at large pore diameters, there is a divergence between the calibrations for pore diameters below about $100 \AA$.

Keywords : NMR, cryoproometry, porosimetry, neutron scattering, SANS.
\end{abstract}

\section{Introduction.}

NMR cryoporometry ${ }^{1}$ is a comparative technique for measuring pore size distributions, in that many of the thermodynamic constants of the indicator liquids used are only known to low precision. It is commonly calibrated using gas adsorption (which relies on the applicability of the Kelvin equation and appropriate choice of pore geometry). Here we present a refined calibration for water using this technique.

We also investigate the possibility of obtaining a direct measurement of pore diameter and other properties of the porous system using information combined from NMR, density measurements and neutron scattering, by calculating the scattering and other properties of extended models of porous systems ${ }^{2}$.

\section{NMR cryoporometric mea- surements.}

A small crystal melts at a lower temperature than a bulk sample. Cryoporometry uses this as a method of measuring pore size.

A liquid (in this case water) was added to fill the pores in the silica and the sample cooled until all the liquid was frozen. Then the sample was warmed slowly, while measuring the amplitude of the NMR signal from the liquid only, until all the ice was melted.

Samples were made using 10 different porous silicas from various sources, of nominal pore diameter $25 \AA, 40 \AA, 46.3 \AA, 49.3 \AA, 60 \AA, 60 \AA, 100 \AA, 140 \AA$, $171.5 \AA$ and $453 \AA$ (as measured by gas adsorption), making two samples of each silica. 


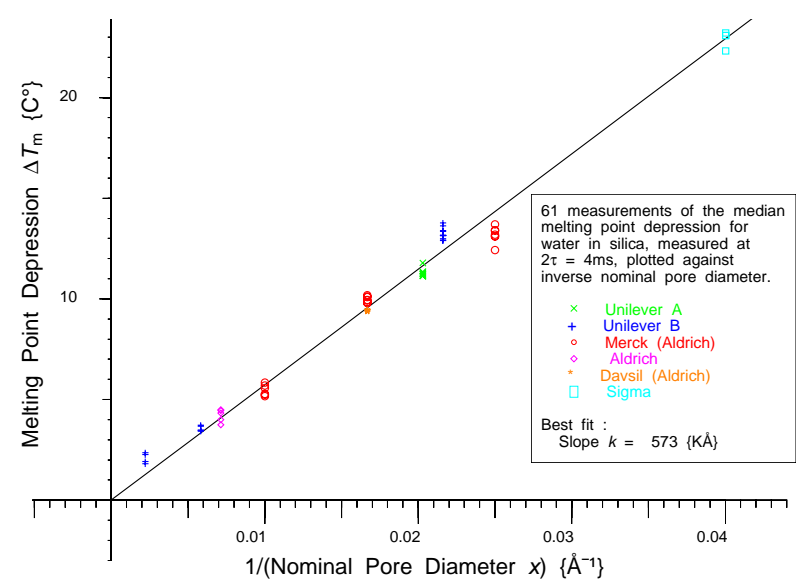

Figure 1: Measured median melting point depression for water in 10 different porous silicas, for $2 \tau=4 \mathrm{~ms}$, plotted against inverse nominal pore diameter.

The amplitude of the NMR echo at time $2 \tau$ was repeatedly measured as a function of the sample temperature, $\tau$ time and warming rate.

For echo time $2 \tau=4 \mathrm{~ms}$, and for a warming rate of $0.5 \mathrm{C}^{\circ} \cdot \mathrm{min}^{-1}$ we obtain the results plotted in figure 1. From this it can be be seen that the six or more measurements for each silica are for the most part self consistent.

If we do a linear least squares fit we obtain a best fit line with a slope of $k=573\{\mathrm{~K} \cdot \AA\}$, where the melting point depression for pore diameter $x$ is given by the simplified Gibbs-Thomson equation $\Delta T_{m}=\frac{k}{x}$.

The results are clustered fairly closely to the best fit line; there is no noticeable deviation even for the $25 \AA$ nominal pore diameter silica. Thus we conclude that at $2 \tau=4 \mathrm{~ms}$ any surface layer effect is having minimal effect. Other workers report a surface layer of $1.75 \AA$ for water ${ }^{3}$.

\section{Density and Imbibation mea- surements.}

Seven of these sol-gel silicas were studied by density and imbibation measurements. The density of each of the porous silicas was measured both dry and with all pore and intergrain void space filled with first water and then with cyclohexane.

Simple measurement of the porous silica density provides information that constrains the range of possible pore geometries within the silica, since the densities of the sol-gel silicas varied by a factor of about three between the large and small pore size silicas.

Additional information obtained by imbibing liquid into the porous sol-gel silicas, and measuring the resultant density includes the (voidless) silica matrix density and grain packing information.

\section{$4 \quad$ Neutron scattering from dry sol-gel silicas.}

Measurements of the intensity of neutron scattering from these seven dry sol-gel porous silicas were performed at the Institut Max von Laue - Paul Langevin (ILL) in Grenoble, using both the D4B high-Q diffractometer and the D22 small angle instrument.

By normalising all measurements to equivalent water scattering, it was possible to combine measurements on D4B with measurements on D22 (at three detector distances), to provide results over an exceptionally wide range of scattering $\mathrm{Q}$ :

$8 \cdot 10^{-4} \AA^{-1} \leq Q \leq 17 \AA^{-1}$. This had the advantage of following the SANS scattering from the porous structure into the diffraction region (figure 2).

The high-Q D22B diffractometer results also gave information on the incoherent scattering intensity from the sample (probably due to surface protons), and by subtracting this scattering, the form factor scattering could be followed down to even lower intensity.

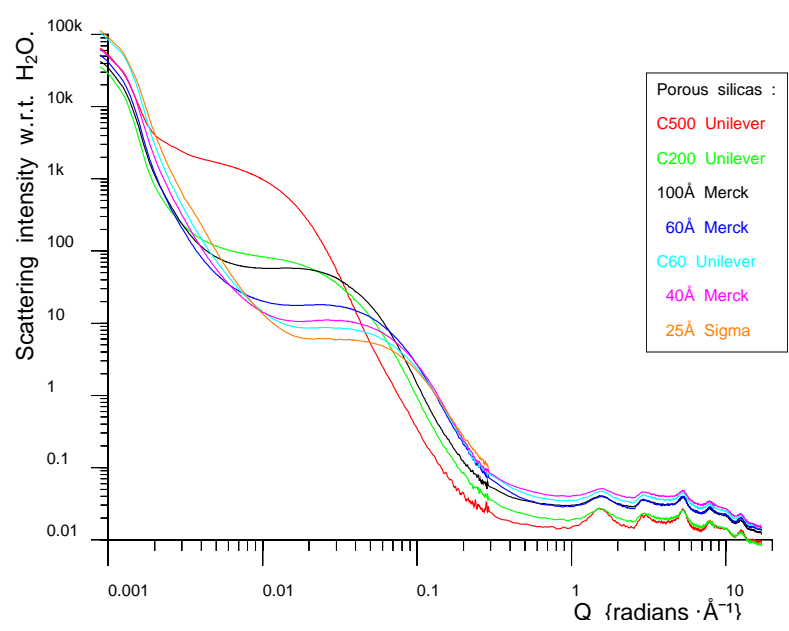

Figure 2: scattering intensity from seven porous silicas, as measured on D22 and D4.

\section{The calculation of the den- sity and the solid-solid den- sity correlation function $G(r)$ for model porous structures.}

Geometric models of porous systems were constructed, both with regular lattices and with variance of lattice and pore diameter, and these were studied by both analytic techniques and Monte-Carlo integration.

The models considered were initially constructed from uniform voids (on regular lattices), excised from a homogeneous medium : firstly cylindrical voids on square lattices and hexagonal lattices and then spherical voids on cubical and hexagonal close packed (HCP) 
lattices, where the voids were permitted to interpenetrate. Analytic techniques were used to study the mean (bulk) properties of the models of the porous structures. Porous models constructed from spherical voids packed on a cubical lattice have a higher density than observed in real sol-gel porous silicas, while the lowest possible density HCP packed voids give densities more typical of aerogels than sol-gel silicas.

By considering analytically the density of these models as a function of the pore diameter to mean lattice spacing ratio $D / a$, it is then possible to deduce by interpolation the density of random packed spherical voids as a function of $D /$ a, without having to explicitly create extended self-consistent random packed models, a non-trivial task.

Comparison of the measured densities for real dry sol-gel silicas and the calculated densities allowed the deduction of values for the pore diameter to mean lattice spacing ratio $D / a$ for the real silicas.

These geometric models were converted to numerical models representing extended porous structures. Monte-Carlo integration of these numeric models then allowed the calculation of solid-solid density correlation functions $G(r)$ and hence the predicted neutron scattering for the models.

$G(r)$ curves were calculated out to 21 times the lattice spacing $a$, with a resolution of $a / 100$. Corresponding calculated neutron scattering curves were over the range $0.0125 / a \leq Q \leq 25 / a$.

Initially regular structures were considered, then ensembles with variance, such that there was a Gaussian distributions of both $D$ and $a$, but, within a given simulation, keeping the ratio $D / a$ constant. The calculated $G(r)$ for the models are then compared with the measured $G(r)$ for the sol-gel silicas, (figure 3), and as seen the results superimpose for each sample for an appropriate choice of just three parameters : pore diameter $D$, pore diameter to lattice spacing ratio $D / a$, and the pore and lattice variance $\sigma$.

\section{The combining of the results.}

Considering the solid-solid density correlation functions $G(r)$ for these sol-gel silicas (figure 3 ), we see that, for appropriate choice of the three parameters pore diameter $D$, pore diameter to lattice spacing ratio $D / a$, and the pore and lattice variance $\sigma$, the calculated curves are in very good agreement with the measured ones. We also note that, in spite of the fact that the best fit models are constructed from spherical voids, that the $G(r)$ curves are very different from those for an isolated sphere of the same size, both in scaling and in shape, due to the interference between the scattering from adjacent voids.

One aim of this work was to investigate the extent that it is possible to use neutron scattering as a direct

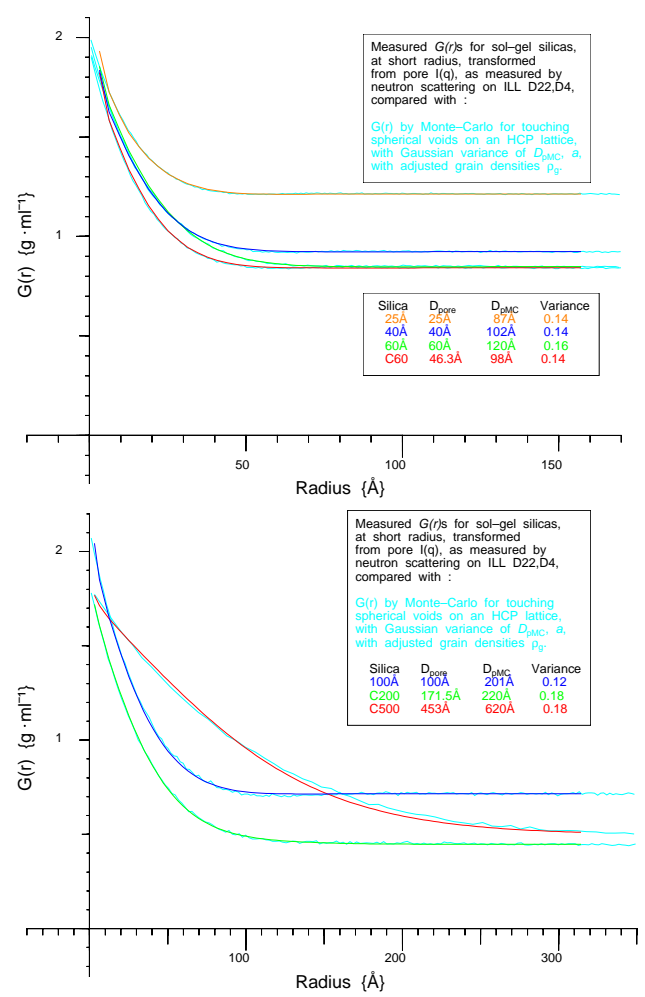

Figure 3:

$G(r)$ for C500 Unilever B, C200 Unilever B, $100 \AA$

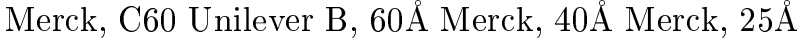
Sigma, $\mathrm{SiO}_{2}$ of nominal pore diameters $D_{\text {pore }}$, at small radius, compared with Monte-Carlo calculation of $G(r)$ for touching spherical voids of diameter $D_{p M C}=$ lattice spacing $a$, on an HCP lattice, with Gaussian variance of $D_{p M C}$, $a$, with average density adjusted to match measured grain densities $\rho_{g}$.

method of determining pore size. If we plot $d(r)=$ $r^{2} \cdot G(r)$, we obtain a function whose peak position may be used to characterise the pore dimension.

It can be shown from both logical argument and from an analysis of the calculated $G(r)$ curves for an exhaustive set of pore models (as a function of the above parameters) that, while the position of the maximum in $d(r)$ can be expected to increase linearly with pore diameter $D$ for an isolated spherical void, it does not necessarily increase linearly (or even monotonically) as the parameter $D / a$ increases.

However, using the models and their calculated $G(r)$ s, a detailed mapping function was generated for the peak position in $d(r)$, as a function of pore diameter $D$, pore diameter to lattice spacing ratio $D / a$, and pore and lattice variance $\sigma$.

The NMR cryoporometry pore diameter measurements for the sol-gel silicas show excellent co-linearity with pore diameters as measured by gas adsorption.

The calculated pore diameters from the measured neutron scattering show surprisingly good agreement 


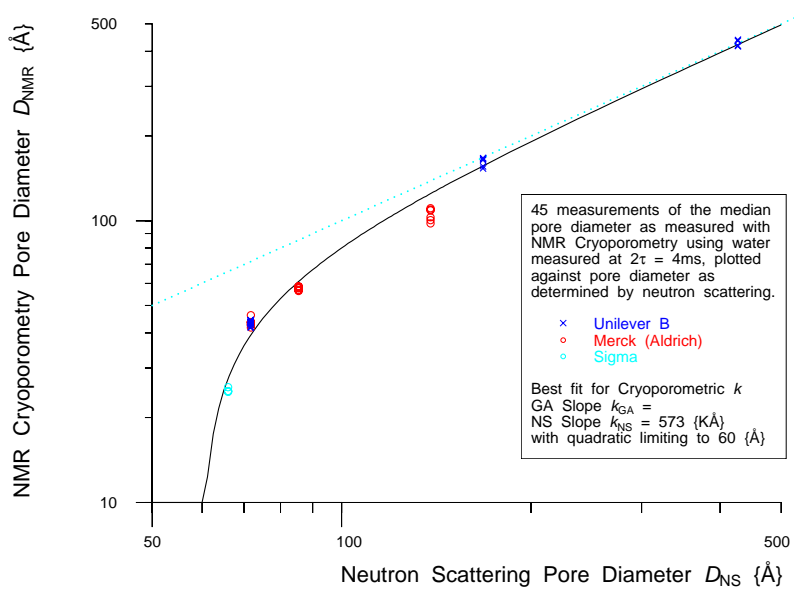

Figure 4: NMR cryoporometric pore diameter vs. that measured by neutron scattering.

with these measurements at large pore diameters. However below about $100 \AA$ there is a divergence between the two calibrations, such that the neutron scattering measurements imply that the pores are much larger than that indicated by both gas adsorption and NMR cryoporometry (figure 4).

This effective 'compression' at small pore size of the separation between the different sol-gel samples can even be seen in the raw neutron scattering curves, on both the small-angle and diffraction results, and is not just a property of the subsequent analysis.

Looking for an explanation for this discrepancy between the techniques, we note that we may expect SANS to give a direct linear scale measurement of structural dimension for constant geometry; we see in the density and imbibation experiments that this geometry changes with pore diameter, the smaller pore sol-gel silicas being denser. However we have exhaustively modeled these changes with pore geometry, and have a function that mimics them. The main remaining limitation is that our model, while it has Gaussian variance of pore diameter $D$ and lattice spacing $a$, nonetheless constrains both to vary together (as we wish to have $D / a$ as a parameter), and we note that this variance is larger than that determined by NMR cryoporometry for the pore size. Thus we conclude that in real sol-gel silicas the lattice spacing variance is probably greater than the pore diameter variance, in contradiction to our modeling assumption. None the less it is not clear how such a modeling error could give rise to such a marked error in deduced pore size, only at small pore sizes.

Turning to the well established gas adsorption calibration, we note that this uses the Kelvin equation to calculate pore size distributions from the desorption $P(v)$ curve, using a pore model of right cylinders.

It is possible that molecular size effects and deviations from the bulk values for the thermodynamic constants may influence calibration at small pore di- ameters. It may be shown that gas adsorption in effect measures the ratio of pore surface area to pore volume; thus the changes in geometry between large and small pore silicas may also influence the gas adsorption calibration.

\section{Conclusions.}

By applying the relations deduced from the models we may use the information from the three sources, NMR cryoporometry, density and imbibation, and neutron scattering, to obtain a very full set of information describing the sol-gel silicas studied.

The synergy that comes from using these multiple techniques results from the fact that while each technique may only provides partial information, or information that is 'blurred' over more than one parameter, the use of these three techniques together provides information that satisfies a complete set of analytic equations that may then be solved.

As a simple example, while density measurements return the SilicaVolume + PoreVolume + InterGrainVolume for a given mass of silica, NMR cryoporometry provides a convenient way to measure the Pore Volume on it's own, and imbibation provides the PoreVolume + InterGrainVolume.

We thus find we may obtain for each of the silicas studied the fully density corrected solid-solid density correlation function $G(r)$ (figure 3), deduction of the density of the (voidless) silica matrix out of which the pores are excised, measurement of the silica fraction in the grain and of the packing fraction of the silica grains and an estimation of the water equivalent residual hydrogen on the dried silica surfaces ${ }^{2}$. In addition we obtain the three parameters pore diameter $D$, pore diameter to lattice spacing ratio $D / a$, and the pore and lattice variance $\sigma$. Full details will be published elsewhere.

The gas adsorption and NMR cryopororometry show good co-linearity of pore size as expected, and the neutron scattering data shows surprisingly good agreement at large pore sizes, but diverge below $100 \AA$.

We hope to perform further simulation work to help understand the neutron scattering data - clearly there is a need to model extended truly random arrays of voids, but this is a non-trivial problem. Larger pore zeolites and templated MCM silicas of known structure may play a useful calibration role in further work at small pore dimensions.

\section{References.}

1. Strange, J.H.; Rahman, M. ; Smith, E.G.. Phys. Rev. Let.; 71; 21:3589-3591; 1993.

2. Webber, J.B.W.; Ph.D. Thesis, University of Kent, Canterbury, U.K.; January 2000.

3. Hansen, E.W.; Stocker, M; Schmidt, R. J. Chem. Phys., 100(6):2195-2200; 1996. 